\title{
Dynamic Invasion Correction Charts for Dual-Induction Logs
}

\author{
Jianhua Zhang \\ Science College, Xi'an Shiyou University, Xi'an, 710065, China \\ jhzhang@xsyu.edu.cn
}

Keywords: Invasion; Resistivity; Saturation; Time

\begin{abstract}
Traditional invasion correction tornado charts for dual-induction log was based on the step-invasion profiles that is different from a realistic invasion process. The dynamic invasion correction charts can be obtained from the dynamic invasion responses of dual-induction logs. The invasion time was introduced as an important parameter during the correction process. The initial water saturation in a reservoir can be directly obtained from the present charts.
\end{abstract}

\section{Introduction}

In petroleum exploration, fluid saturation in a formation is an important parameter for reservoir evaluation, but it cannot be directly measured in situ. It can usually be estimated from the measurement of formation resistivity, which can be obtained by the devices of resistivity logging [1]. Resistivity logging is a primary method to estimate the true formation resistivity. However, the measurements of resistivity instrument responses were significantly influenced by invasion of drilling-mud filtrate into the reservoir adjacent to the borehole [2].

After a formation was opened, the mud was used during well drilling. Then, the mud filtrate pored into the formation and invasion zone was formed. The invasion process has significant effects on resistivity logging devices. The results of resistivity measurement will deviate from the true formation resistivity. Thus, errors were introduced during the estimations of both true-formation resistivity and fluid saturation $[3,4]$.

Traditional method to reduce the invasion effect was to use resistivity invasion correction charts. The invasion correction charts, sometimes referred to as "tornado" or "butterfly" charts[5], are used to define the depth of invasion, the ratio of invasion zone and true resistivity. All assume a stepcontact profile of invasion and that all resistivity measurements have been corrected. The tornado charts based on a step-invasion profile, in which mud filtrate displaces the native fluids in a pistonlike fashion. The front of mud filtrate front is characterized by a step invasion profile [3].

However, the invasion of mud into formation is a time-dependent process [6-9]. First, the boundary of the displacement is not a sharp or distinct step in a real invasion process. Second, invasion profiles of formation and fluids will change with time. These time-dependent invasion profiles lead to the resistivity log response are functions of invasion time. Therefore, the resistivity invasion correction charts need considered the effects of mud invasion time or logging time since the formation was opened. The present work suggested a method to calculate the dynamic resistivity responses for dual-induction $\operatorname{logs}$ and can provide the dynamic resistivity invasion correction charts.

\section{Dynamic Resistivity Invasion Responses}

The present dual-induction logging responses calculation included four steps as following.

First, both formation-water saturation and salinity were calculated using two-phase percolation theory. While drilling mud filtrate invading into a reservoir, movable oil or gas were radially displaced by the filtrate. It was assumed that both the oil and water in formation are immiscible. Gravity was neglected, but capillary forces were accounted for. The radial displacement process in the invasion model was calculated from fluid flow equations [8,9]. The radial distribution of formation water saturation and salinity at time $t$ were calculated with appropriate boundary conditions. 
Then the formation-water resistivity was obtained as a function of formation water salinity and formation temperature $T$ [8].

Third, the time dependent formation resistivity profile, $R_{f}(r, t)$, was obtained using Archie's formula.

Finally, after the resistivity profiles $R_{f}(r, t)$ at a specified time $t$ had been obtained, the dynamic resistivity response of deep induction array was

$$
R_{I D}(t)=\left[\int_{0}^{\infty} \frac{g_{I D}(r)}{R_{f}(r, t)} d r\right]^{-1},
$$

The dynamic resistivity response of medium induction array was

$$
R_{I M}(t)=\left[\int_{0}^{\infty} \frac{g_{I M}(r)}{R_{f}(r, t)} d r\right]^{-1},
$$

Where $g_{I D}$ and $g_{I M}$ were the radial differential geometrical for deep- and medium-induction array, respectively.

More details about the dynamic responses of dual-induction logs can be found in our previous literature [8,9]. Fig.1 and Fig.2 gave an example from the present calculation. The dash line and solid line in Fig. 1 illustrated the relationship of $R_{I M}$ and $R_{I D}$ with invasion time $t$ respectively. These calculations were the case that the fresh mud with low salinity, $10000 \mathrm{mg} / \mathrm{L}$, invaded into a formation with high-salinity native water, $12000 \mathrm{mg} / \mathrm{L}$; and the initial water saturation was 0.5 . Since the ratio of $R_{I D}$ and $R_{I M}$ is important for reservoir evaluation, Fig.2 draw the change of $R_{I D} / R_{I M}$ with time $t$ as well.

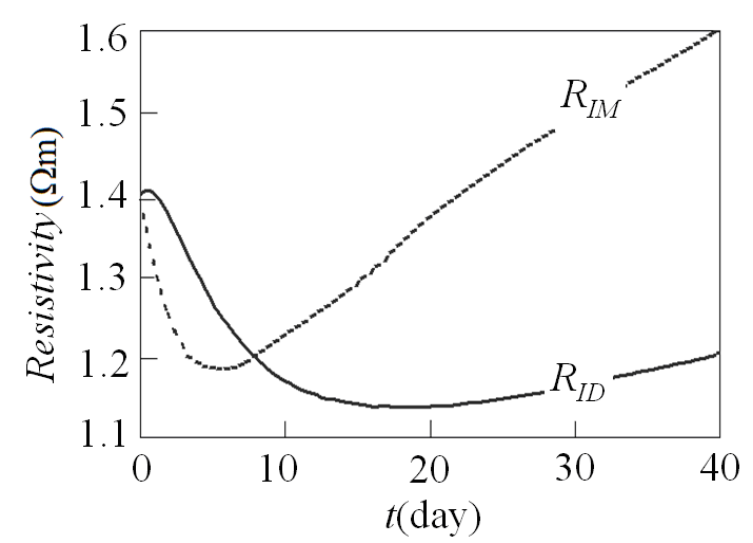

Figure 1. The relationship of $R_{I D}$ and $R_{I M}$ with time $t$

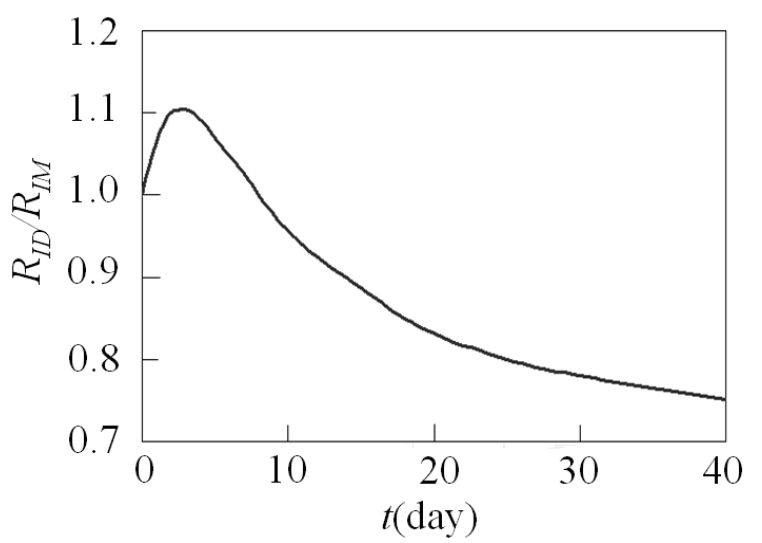

Figure 2. The change of $R_{I D} / R_{I M}$ with time $t$

The dual induction responses at $t=0$ corresponded the moment when the invasion not occurs, so they have the same values that is the true-formation resistivity $R_{t}$. With time lapse, the mud filtrate pored into the formation, the distributions of formation fluids changed with time. The various distributions of formation fluids lead to the resistivity profiles $R_{f}(r, t)$ changed with invasion time. Thus, if a bed was measured using induction logging device at different time, the logging responses would be different. When the bed was measured within one week of drilling, that is the result $R_{I D}>R_{I M}$ was recorded at the early time stage. When the bed was logged after one week of mud filtrate invaded into the formation, $R_{I M}>R_{I D}$ was recorded because the movement of high-resistivity mud filtrate had reached the deep formation and influenced the shallow detective induction array readings $R_{I M}$.

The dynamic invasion process of mud filtrate into a formation has remarkable influences upon the induction logging tools. A dual-induction tool records the responses of both a shallow and a deep focused array simultaneously. The dual-resistivity responses logged early in the invasion process are very different from similar responses logged at later time periods. If the uncorrected resistivity responses are considered to be the formation resistivity and are used to estimate the saturation, error would be introduced. 


\section{The Dynamic Invasion Correction Charts for Dual-Induction Logs}

Traditional resistivity invasion correction charts for dual induction logs was the tornado charts[5] that was based on a step profile of invasion, in which the mud invasion zone was considered as a depth $r_{i}$, from the wellbore and resistivity, $R_{x o}$. The step-profile model differs from the real invasion process. The present dynamic invasion style much agrees with the realistic invasion process. After the calculations of dynamic invasion profiles were calculated, the resistivity responses of mediumand deep-induction logs can be calculated from (1) (2). Furthermore, the time-dependent invasion correction charts for dual-induction logs can be obtained, that is the dynamic invasion correction charts. Fig.3(a) and 3(b) illustrated the dynamic invasion correction charts for fresh mud and saline mud, respectively. The vertical axis was the ratio of deep-induction response $R_{I D}$ and medium reading $R_{I M}$. The horizontal axis was the time after the formation was opened. The main formation parameters, such as mud filtrate salinity $C_{m f}$, water salinity $C_{w}$, formation porosity $\varphi$ and permeability $k$, were marked at the upper right corner in Fig.3.

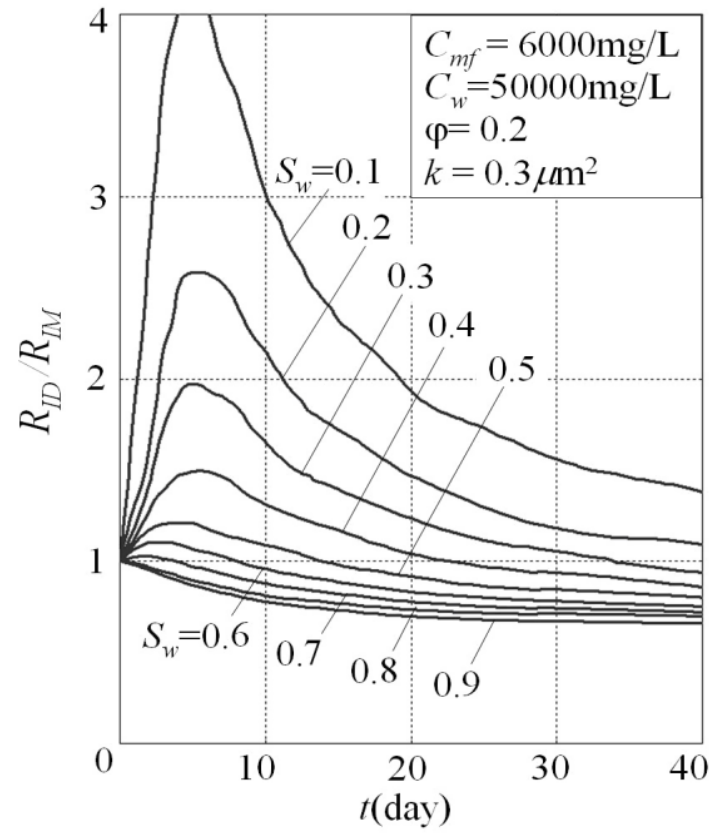

(a)

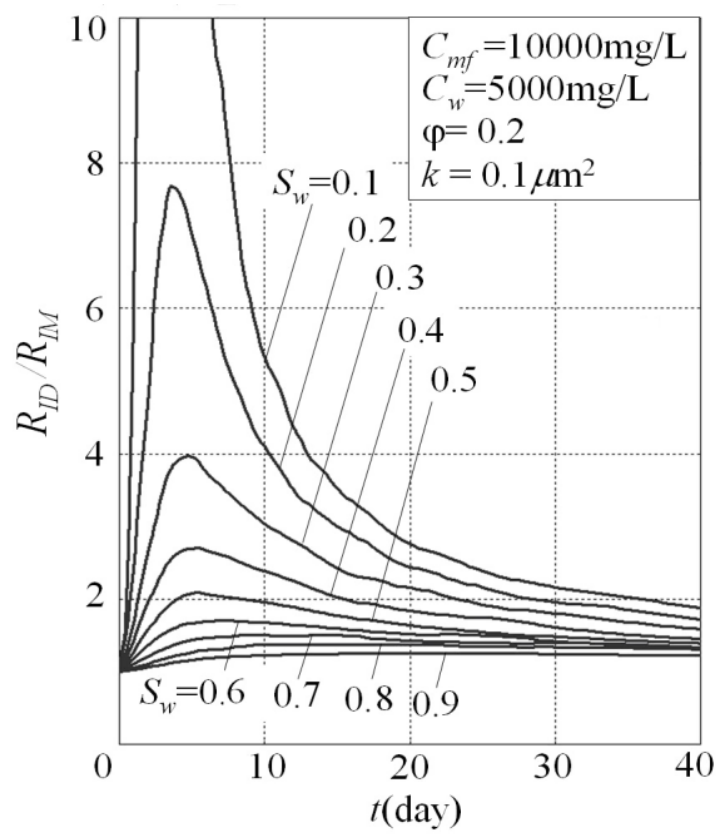

(b)

Figure 3 dynamic invasion correction charts: (a) for fresh mud, (b) for saline mud.

In reservoir evaluation, the ratio of $R_{I D}$ and $R_{I M}$ is an important parameter. While fresh mud was used during drilling, $R_{I D} / R_{I M}>1$ for an oil-bearing reservoir and the value of water saturation $S_{w}$ was low, as shown in Fig.3(a). For a water zone, the ratio, $R_{I D} / R_{I M}<1$, water saturation $S_{w}$ has greater value that is close to 1 .

If a formation contains both oil and water, the water saturation $S_{w}$ has the medium value between 0 and 1. For this situation, a low resistivity annulus appeared in the front of invaded zone [6-8]. It leads the measured data of dual-induction logging alternated with time. When the zone in the neighbor of borehole was filled the high saturation mud filtrate and the saturation in this region was high. With the lapse of time, the filtrate moved into the deep formation. At the early time stage, the result $R_{I D}>R_{I M}$ was recorded since the medium induction array response is focused mainly in the low resistivity annulus. With the time lapse, this low-resistivity annulus had migrated to the response region of the deep-induction array and the high-resistivity mud filtrate had migrated into the medium-induction response region. Thus, $R_{I M}<R_{I D}$ was recorded. When the low-resistivity annulus had migrated to the response region of the deep-induction array and the high-resistivity mud filtrate had migrated into the medium-induction response region, $R_{I M}>R_{I D}$ was recorded at later time stage, as these curves marked $S_{w}=0.4,0.5,0.6$ and 0.7 in Fig.3(a). 
The present dynamic invasion resistivity correction chart is helpful for reservoir. For the formation parameters in Fig.3(a), if a well was logged at 5days of drilling and the value of $R_{I D} / R_{I M}$ is roughly equal to 2, the chart in Fig.3(a) suggests that the initial water saturation $S_{w}$ is 0.3 . Therefore the bed is mainly filled by oil.

Fig.3(b) is the case for low-resistivity saline mud filtrate invading a reservoir. For saline mud invasion, the resistivity in the adjancy of wellbore is low. The shallow detective medium-induction array give less reading and deep-induction log give greater response; thus $R_{I D}>R_{I M}$ was greater than 1 for all invasion period. The curves of $R_{I D} / R_{I M}$ with time for various water saturation $S_{w}$ can also be used to correct the invasion effect.

\section{Conclusions}

The traditional invasion correction tornado charts was based on step invasion model that is different from a realistic invasion process. In order to avoid the mistake estimation during reservoir evaluation, the dynamic invasion style is a suitable model in invasion correction.

The method of dynamic invasion correction consider the invasion time as an important parameter during the correction process. It can directly suggest the initial water saturation in a reservoir.

The present dynamic invasion correction charts for dual-induction tool provides a new, more realistic method for formation evaluation to evaluate oil or water reservoirs. It also can be used to other logging tools.

\section{References}

[1] J. Tittman, Geophysical Well Logging, Academic Press, Inc., Orlando, p82, 1986

[2] A. Y. Windarto, Gunawan, P. Sukarno, E. Soewono, "Modeling of mud filtrate invasion and damage zone formation", J. Pet. Sci. Eng. Vol. 77 (2011) No.3-4, p359-364

[3] Howard A.Q., A new invasion model for resistivity log interpretation, The Log Analyst, Vol. 33 (1992) No.2, p96 110

[4] Akinsete, Oluwatoyin O. and Adekoya, Doyinsola A., Effects of mud filtrate invasion on well log measurements, SPE Nigeria Annual International Conference and Exhibition 2016, August 2-4, 2016

[5] R. M. Bateman and C. E. Konen, Log Anlyst and The Programmable Pocket Calculator - 4. Dual Induction-Laterolog 8, The Log Analyst, Vol. 19 (1978) No.3, p3-7

[6] D. P. Tobola and S. A. Holditch, "Determination of reservoir permeability from repeated induction logging", SPE Formation Evaluation, p20-27, March, 1991

[7] C. Y. Yao and S. A. Holditch, "Reservoir permeability estimation from time-lapse log data", SPE Formation Evaluation, p69-74, June, 1996.

[8] J. H. Zhang, Q. Hu and Z. H. Liu, "Estimation of true formation resistivity and water saturation with a time-lapse induction logging method", The Log Analyst, Vol. 40 (1999) No.2, p38-148

[9] Z. H. Liu, J. Oyang, and J. H. Zhang, "Dynamic Dual-Laterolog Responses: Model and Field Applications in the Bohai Gulf of China", Journal of Petroleum Science and Engineering, Vol. 23 (1999) No.1-2, p1-11 